\title{
Tocilizumab in the treatment of systemic-onset juvenile idiopathic arthritis - single-centre experience
}

\author{
Justyna Roszkiewicz, Krzysztof Orczyk, Elżbieta Smolewska \\ Department of Paediatric Cardiology and Rheumatology, Medical University of tódź, Poland
}

\begin{abstract}
Objectives: The aim of the study was to evaluate the efficacy and long-term safety of tocilizumab treatment in children with systemic-onset juvenile idiopathic arthritis in a single centre.

Material and methods: The study was based on a retrospective analysis of a cohort of 10 patients with systemic-onset juvenile idiopathic arthritis who were treated with tocilizumab in the period September 2011-July 2017. Their medical records were analysed taking into consideration the effectiveness of tocilizumab treatment and frequency of side effects.

Results: Before the initiation of treatment, $9 / 10$ patients from the study group complained of fever and had significantly increased values of inflammatory markers, with the median CRP concentration $41.1 \mathrm{mg} / \mathrm{l}$ (norm $<5 \mathrm{mg} / \mathrm{l}$ ) and ESR $37 \mathrm{~mm} / \mathrm{h}$ (norm $<12 \mathrm{mg} / \mathrm{l})$. The period of the initial 12 weeks of treatment was a quantum leap in the course of the disease: all children were afebrile, and inflammatory markers values decreased by $99.4 \%$ in the case of CRP and $91.9 \%$ in ESR. All patients fulfilled ACR Pedi 50 criteria, and 3 of them achieved ACR Pedi 70. In the next stages of treatment the response to tocilizumab was sustained, reaching 10 children achieving ACR Pedi 70 and 5 ACR Pedi 90 after one year of therapy. Tocilizumab appeared to be relatively safe in the study group. Although elevation of transaminases and neutropenia were observed in 5/10 patients, they were usually mild and transitional in their course.

Conclusions: Tocilizumab is both effective and has a relatively good safety profile in children with severe systemic-onset juvenile idiopathic arthritis. It should be considered in the recommendations as a first-line treatment of this disease.
\end{abstract}

Key words: tocilizumab, systemic-onset juvenile idiopathic arthritis, treatment response, biologic treatment effectiveness.

\section{Introduction}

Systemic-onset juvenile idiopathic arthritis (s)IA) is the most severe and distinctive subtype of juvenile idiopathic arthritis (JIA). In contrast to other JIA subtypes, the clinical course of $S J I A$ is characterised by prominent features of systemic inflammation: spiking fever, macular rash, hepatosplenomegaly, lymphadenopathy and serositis in combination with arthritis. Laboratory test results show remarkably high values of acute phase proteins such as C-reactive protein (CRP), erythrocyte sedimentation rate (ESR) and ferritin, and a shift in blood morphology: leukocytosis with predominant neutrophilia and thrombocytosis [1].

The presence of systemic manifestations is suggested to be caused by continuous activation of innate immunity pathways leading to dysregulated production of proinflammatory cytokines, such as IL-1 $\beta$ and IL-6 [2]. Treatment of SIIA is challenging. The systemic features during the flare of the disease are very severe, and there is a possibility of developing macrophage activation syndrome (MAS), a potentially life-threatening complication of sJIA. Before the introduction of biologic treatment, patients usually required long-term administration of 
systemic steroids in high doses, which could lead to numerous side effects of the therapy such as growth delay, osteoporosis and hypertension. Efficacy of many disease-modifying antirheumatic drugs (DMARDs) such as methotrexate (MTX) and tumour necrosis factor $\alpha$ inhibitors (anti-TNFs) is lower in SJIA than in other JIA subtypes [3-5].

Tocilizumab (TCZ) is a humanized monoclonal antibody that acts as a receptor-inhibitor of IL-6. IL-6 is a cytokine possessing multiple biologic functions that are important in inflammation: it induces hepatocytes to produce acute-phase proteins, promotes osteoclast activation, increases angiogenesis and induces fever generation. IL-6 contributes to B-cell and T-cell differentiation and inhibition of regulatory T-cell production. Levels of IL-6 are significantly higher in sera of JIA patients than in healthy controls [6]. Registration of biologic agents targeting IL- 6 was a milestone in the treatment of sJIA and nowadays inactive disease and remission are within reach of most patients. According to the Polish guidelines [7], TCZ is indicated in children with sJIA over the age of two who fulfil one of the clinical criteria:

- sJIA with predominant systemic features, not responding adequately to at least a two-week course of systemic steroids in the dose $1-2 \mathrm{mg} / \mathrm{kg} /$ day of oral prednisone or $10-30 \mathrm{mg} / \mathrm{kg} /$ day of intravenous methylprednisolone,

- sJIA with involvement of at least five peripheral joints or two joints and coexisting fever, in whom the disease is active despite at least three-month treatment with systemic steroids and MTX or another immunosuppressive drug.

The aim of our study was to evaluate the efficacy and safety of tocilizumab treatment in all children with systemic-onset juvenile idiopathic arthritis in our department. To our knowledge, this is the first study on that topic in the Polish population.

\section{Material and methods}

Medical histories of SJIA patients treated with TCZ in our department between September 2011 and April 2017 were retrospectively analysed. The diagnosis of sJIA was based on the International League of Associations for Rheumatology (ILAR) classification criteria of JIA [8]. The dose of TCZ was $12 \mathrm{mg} / \mathrm{kg} /$ dose every 2 weeks in children weighing under $30 \mathrm{~kg}$ and $8 \mathrm{mg} / \mathrm{kg} /$ dose every 2 weeks in children weighing over $30 \mathrm{~kg}$. Patient demographic data, including age, gender, clinical manifestations of the disease, duration of symptoms and medications used in the history, were collected at the initiation of TCZ treatment. Laboratory test results included in the study were obtained before initiation of TCZ treatment, after one dose of TCZ and after 3, 6 and 12 months of drug administration. The treatment response was evaluated at 3, 6 and 12 months of treatment using American College of Rheumatology Paediatric criteria (ACR Pedi).

Medical records of patients were also analysed for treatment side effects, including leukopenia, hepatotoxicity and opportunistic infections during the first two years of the therapy. Baseline characteristics, treatment response, and ACR Pedi 30, 50, 70 were calculated using simple descriptive statistics. Spearman rank correlation analysis between age at diagnosis, baseline inflammatory markers values, time from disease onset to TCZ treatment initiation and time to achieve ACR 30, 50, 70 criteria was performed. The data were analysed using Statistica 13 software (StatSoft Polska, Kraków, Poland).

\section{Results}

\section{Group baseline characteristics}

The study group was composed of 10 patients: 6 girls and 4 boys diagnosed with sJIA aged from 2 to 15 years (mean age $7.11 \pm 2.06$ years). Nine of the patients were febrile at the baseline of treatment, and in 6 of them we found a limited range of motion within peripheral joints with coexistence of oedema in 3 patients. The median time of TCZ treatment initiation was 167 days. The first patient was administered TCZ 4.5 years after SJIA diagnosis, the last one after 23 days. This shift probably reflects progress in access to biologic treatment in Poland. At the baseline of TCZ treatment all patients from the study group received systemic steroids and DMARDs: MTX in 9 patients, cyclosporine in 1. Three of them were previously treated with a biologic DMARD - etanercept in their medical history.

Before administration of the first TCZ dose, we observed increased values of inflammatory markers in 9 out of 10 patients. Their median values were as follows: CRP $41.1 \mathrm{mg} / \mathrm{l}$ (range 4.96-182.0), ESR $37 \mathrm{~mm} / \mathrm{h}$ (range 3-70). The disease activity was measured using the VAS analogue scale assessed both by parent of the patient and their physician. Their median values were $75 \mathrm{~mm}$ (range 40-100) and $40 \mathrm{~mm}$ (range 30-60), respectively. Median value of the Childhood Health Assessment Questionnaire (CHAQ) was 1.275 (range 0.5-1.4) (Table I).

\section{Treatment effectiveness after 12 weeks}

At 12-week assessment none of the patients complained of fever. The number of children who achieved ACR Pedi 30, 50 and 70 at this time point was 10, 10 and 3 , respectively (Fig. 1). At this time point all of the children reached the state of clinically inactive disease according to Wallace criteria [9]. In all patients we ob- 
Table I. Patient baseline characteristics

\begin{tabular}{|lc|}
\hline Characteristic & Patients $(N=10)$ \\
\hline Female gender & 6 \\
\hline Age (in years) & $7.11 \pm 2.06$ \\
\hline Duration of disease (in days) & 330 \\
\hline Prior use of DMARDs & 10 \\
\hline Methotrexate & 9 \\
\hline Cyclosporin A & 1 \\
\hline Prior use of a biologic agent & 3 \\
\hline Prior use of anti-TNF agent & 3 \\
\hline Prior use of IL-1 inhibitor & 0 \\
\hline Oral glucocorticoid use & 10 \\
\hline
\end{tabular}

Plus-minus values are means $\pm S D$

$D M A R D$ - disease-modifying antirheumatic drug; TNF - tumour necrosis factor

served a significant decrease of inflammatory marker values. The median CRP value was $0.225 \mathrm{mg} / \mathrm{l}$ (range 0.1-1.56). Absolute change of CRP concentrations from baseline was $-40.775 \mathrm{mg} / \mathrm{l}$, and median percentage change was $-99.4 \%$. The median ESR value at the same time point was $3 \mathrm{~mm} / \mathrm{h}$ (range $2-12$ ), which means the absolute median change from baseline was $-34 \mathrm{~mm} / \mathrm{h}$, and the median percentage change was $-91.9 \%$.

In 5/10 patients we observed a decrease in inflammatory marker values to normal limits after infusion of one TCZ dose (Table II). After 12 weeks of TCZ therapy all of the children were still receiving oral corticosteroids, but in 9 of them we were able to reduce their doses by at least 30\%.

\section{Treatment effectiveness after 24 weeks}

After 6 months of therapy the number of children who achieved ACR Pedi 30, 50, 70 had improved and reached 10, 10 and 8, respectively. Three patients achieved ACR Pedi 90 (Fig. 1). None of the children complained of joint stiffness, and peripheral joint oedema was not noted. The values of inflammatory markers remained within normal limits. The median CRP value was $0.2 \mathrm{mg} / \mathrm{l}$ (range 0.14-1.36), which means no significant change in this parameter between 3 and 6 months of treatment (absolute median change - $0.025 \mathrm{mg} / \mathrm{l}$, percentage change $-11.2 \%$ ). The same observation applied to ESR. Median ESR value was $2 \mathrm{~mm} / \mathrm{h}$ (range 2-10), which corresponds to the absolute median change of -1.0 , which is not clinically significant. Due to satisfactory treatment results in half of patients, systemic corticosteroids were discontinued, and in the remaining half their doses were gradually tapered down.

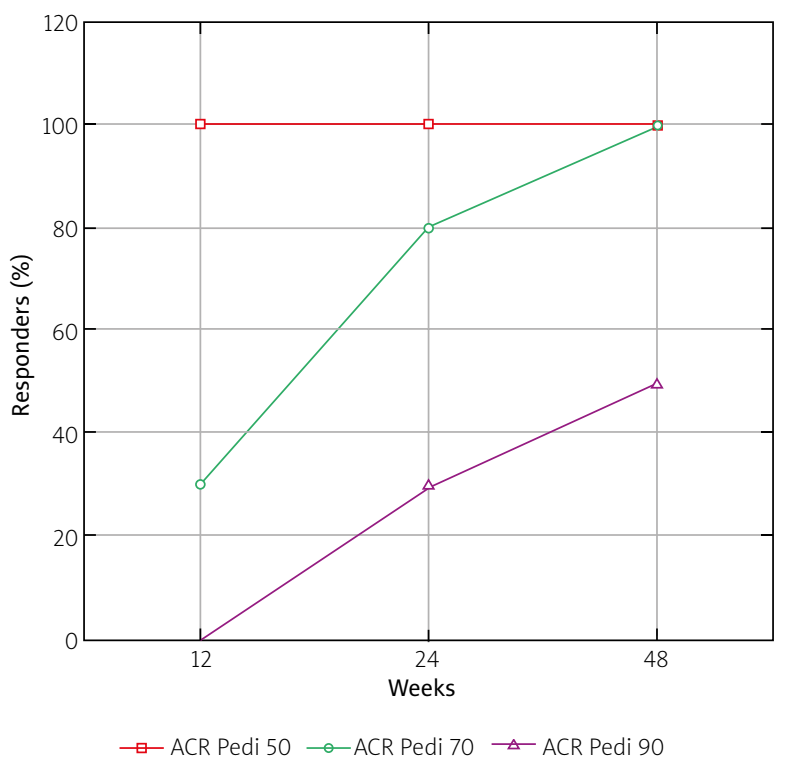

Fig. 1. Time courses of American College of Rheumatology Pediatric (ACR Pedi) 30, 50, and 70 responses.

\section{Treatment effectiveness after 48 weeks}

At 12 months of treatment we were able to analyse 8 of the patients from the study group, as 2 of them had not completed one year of the treatment period yet. Only 2/8 patients were still receiving oral corticosteroids at low doses at this time point. In 1 patient MTX was discontinued and TCZ was used in monotherapy without aggravation of symptoms. At 48 weeks of TCZ treatment all of the patients had achieved ACR Pedi 30, 50 and 70, and half of them had reached ACR Pedi 90 (Fig. 1). The values of inflammatory markers were within normal limits. There was no change in CRP median concentration between 24 and 48 weeks of treatment $(0.2 \mathrm{mg} / \mathrm{l}$, range 0.1-0.38), and ESR was also at the same level (median $2.5 \mathrm{~mm} / \mathrm{h}$, range $2-6)$.

We found no significant correlations between duration of the disease before the initiation of TCZ treatment, age of the patient, baseline values of inflammatory markers and the clinical outcome of TCZ treatment, measured as time to achieve ACR Pedi criteria (Spearman's rank correlation $R$-values for ACR Pedi 70 response: $\left.R_{E S R}=0.07, R_{C R P}=-0.12, R_{\text {age }}=-0.04, R_{\text {time }}=0.23\right)$.

\section{Adverse effects of the therapy}

Mild upper respiratory tract infections, which led to omitting one dose of TCZ, were reported with the frequency 0.4 infection/patient-year. No tuberculosis or Pneumocystis jiroveci opportunistic infection was noted. 
Table II. Change from Baseline in ACR Core Set of Variables

\begin{tabular}{|lcccc|}
\hline Variable & Baseline & Week 12 & Week 24 & Week 48 \\
\hline JIA ACR 70 response and no fever $(n)$ & - & $3(30 \%)$ & $8(80 \%)$ & $10(100 \%)$ \\
\hline ACR core set variables & & & & \\
\hline No. of patients with active arthritis & 3 & 0 & 0 & 0 \\
\hline No. of patients with limited range of motion & 6 & 0 & 0 & 0 \\
\hline Score for physician's global assessment of disease activity & $40^{*}$ & $10^{*}$ & $5^{*}$ & $0^{\star}$ \\
\hline Score for patient's global assessment of overall wellbeing & $75^{*}$ & $35^{*}$ & $20^{*}$ & $15^{*}$ \\
\hline CHAQ score & $1.275^{*}$ & $0.675^{*}$ & $0.55^{*}$ & $0.375^{*}$ \\
\hline ESR $(\mathrm{mm} / \mathrm{h})$ & $37^{*}$ & $3^{*}$ & $2^{*}$ & $2.5^{*}$ \\
\hline
\end{tabular}

* values are medians

Herpes simplex type 1 infection was rare (0.11/patient-year) during the period of TCZ treatment.

In half of patients neutropenia was observed, which corresponds to 0.77 incident/patient-year. In all cases it was transitional, grade 1 neutropenia, described as an absolute neutrophil count $<2.0 \times 10^{9} /$ and $>1.1 \times 10^{9} /$. The median TCZ dose received before the first episode of neutropenia was 10 (range 5-15). Increase in aspartate aminotransferase and alanine aminotransferase activities was the most common side effect of TCZ treatment (3.33 episodes/patient-year). All the patients who developed hepatotoxicity were simultaneously treated with MTX. However, in only 1 patient liver function test values were three times above the normal limits (grade 2 of hepatic toxicity) and treatment with TCZ was suspended until their normalisation. There were no infusion-related treatment adverse effects in our study group. None of the patients developed MAS.

\section{Discussion}

Introduction of TCZ is considered as a breakthrough in the management of SJIA. TCZ shows both high effectiveness and a satisfactory drug safety profile, which makes it an invaluable treatment option even in monotherapy [6], especially in patients resistant to other DMARDs and with high doses of corticosteroid dependency.

This study confirmed both TCZ features, despite the presence of limitations related to retrospective analysis of the data and small study group size. In our patients the most spectacular differences in disease course were observed in the initial 12-week phase of treatment. At this time point none of the children had fever and the values of inflammatory markers decreased significantly, reaching $-99.4 \%$ and $-91.9 \%$ in concentrations of CRP and ESR, respectively.

Moreover, all of the patients reached the state of clinically inactive disease according to Wallace criteria [9] and achieved an ACR Pedi 50 response, with 3 of them reaching ACR Pedi 70. Those results show a similar therapy outcome regarding ACR Pedi 30 and ACR Pedi 50 in comparison to the TCZ therapy response rates achieved in phase III of the randomised, double-blind, placebo-controlled study by Yokota et al. [10], where ACR Pedi 30, 50 and 70 were achieved by 80,80 and $75 \%$, respectively. The difference in achieving ACR 70 criteria at this time point is likely to be affected by the disproportionally high CHAQ values in our study group and the baseline characteristics of our patients, such as relatively low presence of symptoms of arthritis in the course of SJIA (3/10 patients). On the other hand, our outcomes of ACR Pedi 70 and 90 at this time point are comparable with data from the BIKeR study [5], in which after 3 months of treatment ACR Pedi 70 and 90 were reached by $44 \%$ and $16 \%$ of patients, respectively.

In 2005, Woo et al. [11] described achievement of ACR Pedi 30 response criteria in $73 \%$ of sJIA patients after a single TCZ dose. Due to missing data, calculating the ACR 30 criteria response after one dose of TCZ was unachievable. However, we observed absence of fever in all of the patients and normalisation of inflammatory marker values in half of them after one TCZ infusion. As described in previous studies on TCZ efficacy and safety, the response to $T C Z$ is sustained in the following oneyear treatment period [12].

In our study group, after 48 weeks of therapy, all patients achieved ACR Pedi 70 and half of them ACR Pedi 90 criteria. Despite the limited study group size, those outcomes are comparable with results obtained by Yokota et al. [10], where ACR Pedi 30, 50, 70 response rates were $98 \%, 94 \%$ and $90 \%$ and similar to the TENDER study [13], where $87.4 \%$ of patients achieved ACR Pedi 70 after 52 weeks of treatment. In contrast, the results of all the studies mentioned above are divergent from BIKeR registry data [5], in which after 24 months of therapy ACR Pedi 70 and 90 were reached by 40 and $35 \%$ of children. In our patients effectiveness of TCZ treatment 
was not influenced by duration of the disease and disease features at study entry, similarly as observed in the TENDER study. Tapering down the doses of systemic corticosteroids may also be perceived as an indicator of TCZ therapy response. In the TENDER study $61 \%$ of patients discontinued steroids after two years of TCZ treatment [14]. In our population this percentage reached $75 \%$ after the initial 48 weeks of treatment and TCZ proved to be effective even in monotherapy.

Tocilizumab is described as the biologic agent with the highest risk of therapy side effects $[5,15,16]$, with serious adverse effects occurring more frequently in sJIA than in the polyarticular subtype of the disease [6]. In our study group the most common side effects were elevations of liver enzyme activities and neutropenia. Despite the high incidence, both of them were transitional and mild in course. Neutropenia in all cases resolved spontaneously, as observed previously in TENDER and the study by Yokota et al. [10, 14], when it appeared in $15 \%$ and $17.9 \%$ of children, respectively. It is important to note that in the studies mentioned above patients had grade 3 neutropenia (classified as a neutrophil count $\geq 0.5$ to $<1.0 \times 10^{9} /$ ), whereas in our population we observed only grade 1 , defined as a neutrophil count $<2.0$ $\times 10^{9} /$ and $>1.1 \times 10^{\%} /$. Elevated transaminases in our study group were observed in half of the cases, although only 1 patient had grade 2 of this side effect (classified as transaminase activity $>3-5$ times more than the upper limit of normal value) and required temporary suspension of treatment. In other patients values of liver enzyme activities after hepatoprotective treatment decreased to values within normal limits. In previous studies on TCZ, infections were described as the most frequent adverse effect of the therapy. In our population infections occurred with an incidence 0.4 infection/patient-year. In the TENDER study this rate reached 3.0/patient-year and $0.115 /$ patient-year in reference to severe diseases. Such a considerable difference between our study group and TENDER is probably the consequence of retrospective analysis of the data and missing information about infections that children faced between the TCZ infusions that resolved spontaneously and did not result in omitting a TCZ dose.

\section{Conclusions}

Treatment of rheumatic diseases has significantly changed during the last decade. The emerge of biologic drugs has contributed to that fact and made remission or clinically inactive disease within reach of most JIA patients. Tocilizumab is efficacious in severe cases of SIIA, refractory to other therapy. Despite the acceptable safety profile of the drug, the possible treatment bene- fits in every patient must be weighted against the risk of adverse effects of therapy that is higher in TCZ than in other biologic drugs. Our study should be followed by a multicenter prospective analysis in order to evaluate the real safety of TCZ treatment and its potential as a first-line therapy in sJIA.

The study was supported by grant No. 503/8-00004/503-81-002-17 from the Medical University of tódź, Poland.

The authors declare no conflict of interest.

\section{References}

1. De Benedetti F, Brunner HI, Ruperto N, et al. Randomised trial of tocilizumab in systemic juvenile idiopathic arthritis. N Engl J Med 2012; 367: 2385-2395.

2. Cimaz R. Systemic-onset juvenile idiopathic arthritis. Autoimmun Rev 2016; 15: 931-934.

3. Prince $F H$, Twilt $M$, ten Cate $R$, et al. Long-term follow-up on effectiveness and safety of etanercept in juvenile idiopathic arthritis: the Dutch national register. Ann Rheum Dis 2009; 68: 635-641.

4. Horneff G, De Bock F, Foeldvari I, et al. Safety and efficacy of combination of etanercept and methotrexate compared to treatment with etanercept only in patients with juvenile idiopathic arthritis (IIA): preliminary data from the German JIA Registry. Ann Rheum Dis 2009; 68: 519-525.

5. Horneff G, Schulz AC, Klotsche J, et al. Experience with etanercept, tocilizumab and interleukin-1 inhibitors on systemic onset juvenile idiopathic arthritis patients from the BIKER registry. Arthritis Res Ther 2017; 19: 256.

6. Turnier JL, Brunner HI. Tocilizumab for treating juvenile idiopathic arthritis. Expert Opin Biol Ther 2016; 16: 559-566.

7. Program leczenia reumatoidalnego zapalenia stawów i młodzieńczego idiopatycznego zapalenia stawów o przebiegu agresywnym [online]. Narodowy Fundusz Zdrowia, 2017 [Access: 5.08.2018]. Available at: htps://www.gov.pl/ documents/292343/436711/b.33.-nowyod11.2017.docx/ 7ce53e37-6ef9-d6a2-0143-7385f7011713.

8. Petty RE, Southwood TR, Manners P, et al. International league of associations for rheumatology classification of juvenile idiopathic arthritis: second revision, Edmonton, 2001. J Rheumatol 2004; 31: 390-392.

9. Wallace CA, Giannini EH, Huang $\mathrm{H}$, et al. American College of Rheumatology provisional criteria for defining clinical inactive disease in select categories of juvenile idiopathic arthritis. Arthritis Care Res (Hoboken) 2011; 63: 929-936.

10. Yokota S, Imagawa T, Mori M, et al. Efficacy and safety of tocilizumab in patients with systemic-onset juvenile idiopathic arthritis: a randomised, double-blind, placebo-controlled, withdrawal phase III trial. Lancet 2008; 371: 998-1006.

11. Woo P WN, Prieur AM, Southwood T, et al. Open label phase II trial of single, ascending doses of MRA in Caucasian children with severe systemic juvenile idiopathic arthritis: proof of principle of the efficacy of IL- 6 receptor blockade in this type 
of arthritis and demonstration of prolonged clinical improvement. Arthritis Res Ther 2005; 7: R1281-1288.

12. Yokota S, Itoh Y, Morio T, et al. Tocilizumab in systemic juvenile idiopathic arthritis in a real-world clinical setting: results from 1 year of postmarketing surveillance follow-up of 417 patients in Japan. Ann Rheum Dis 2016; 75: 1654-1660.

13. De Benedetti F, Brunner HI, Allen R, et al. The efficacy of tocilizumab in patients with systemic juvenile idiopathic arthritis: 52-week data from a phase 3 clinical trial. In: British Society of Rheumatology Annual Meeting, May 2012.
14. De Benedetti F, Brunner H, Ruperto N, et al. FRI0328 Efficacy and safety of tocilizumab (TCZ) in patients with systemic juvenile idiopathic arthritis (SIIA): 2-year data from tender, a phase 3 clinical trial. Ann Rheum Dis 2013; 71: 425.

15. Horneff G. Biologic-associated infections in pediatric rheumatology. Curr Rheumatol Rep 2015; 17: 66.

16. Tarp S, Amarilyo G, Foeldvari I, et al. Efficacy and safety of biological agents for systemic juvenile idiopathic arthritis: a systemic review and meta-analysis of randomized trials. Rheumatology (Oxford) 2016; 55: 669-679. 